\title{
High-Frequency Whisker Vibration Is Encoded by Phase- Locked Responses of Neurons in the Rat's Barrel Cortex
}

\author{
Tobias A. S. Ewert, Christiane Vahle-Hinz, and Andreas K. Engel \\ Department of Neurophysiology and Pathophysiology, University Medical Center Hamburg-Eppendorf, 20246 Hamburg, Germany
}

\begin{abstract}
Rats perform texture discrimination during tactile exploration with their whiskers with high spatial and temporal precision. Although the peripheral mechanoreceptors provide tactile information with exquisite temporal resolution, physiological studies have suggested that this information might be lost at the cortical level. To address this discrepancy, multiunit and single-unit recordings were performed in the barrel cortex of isoflurane-anesthetized rats using continuous sinusoidal vibration of single whiskers at 15-700 $\mathrm{Hz}$. In multiunit recordings, sustained phase-locked responses occurred up to vibration frequencies of $700 \mathrm{~Hz}$, and 1:1 stimulus locking was observed up to $320 \mathrm{~Hz}$. Wide-band responses of multiunits showed frequency encoding between $20 \mathrm{and} 320 \mathrm{~Hz}$. The discharge rates were not different for stimuli in the low- and high-frequency ranges, but they were significantly lower for non-phase-locked responses to high-frequency vibration. Response adaptation was present in only $25 \%$ of the cases, whereas in the majority of cases, entrainment to the vibratory frequency remained constant or even increased with stimulus duration. Increased entrainment to high-frequency stimulation was accompanied by the emergence of induced activity in the gamma-band range. Analysis of single-unit activity revealed that phase locking to vibratory stimuli was more precise than that observed for the multiunit responses. The results show that whisker vibrations at frequencies above $100 \mathrm{~Hz}$ are faithfully encoded by sustained phase-locked responses of cortical neurons under isoflurane anesthesia. It is conceivable that the awake animal makes use of the tactile signals at even much higher frequencies, which can be provided by the peripheral mechanoreceptors during texture discrimination.
\end{abstract}

Key words: somatosensory system; barrel cortex; gamma oscillations; phase-locking; tactile discrimination; isoflurane anesthesia

\section{Introduction}

Rats use the whiskers arranged around their snout to probe objects and to discriminate among very fine textures (Guic-Robles et al., 1989, 1992; Carvell and Simons, 1990, 1995; Brecht et al., 1997; Krupa et al., 2001; Stüttgen et al., 2006). Whiskers touching rough surfaces are estimated to vibrate at frequencies in the order of $1000 \mathrm{~Hz}$ (Mehta and Kleinfeld, 2004) and were recently demonstrated to engage in high-velocity micromotions (Ritt et al., 2008). They originate in sinus hair follicles that house several types of mechanoreceptors (Andres, 1966; Rice et al., 1997; Ebara et al., 2002) exquisitely sensitive to high-frequency, low-amplitude vibrissal movements. The primary afferent fibers discharge with high precision to stimulus transients (Gibson and Welker, 1983; Shoykhet et al., 2000; Jones et al., 2004a,b) and phase locked to vibrations up to $1 \mathrm{kHz}$ (Deschênes et al., 2003). Mechanoreceptors from vibrissa follicles of cats have been shown to respond precisely phase locked even to much higher frequencies (Gottschaldt and Vahle-Hinz, 1981). Thus, mechanoreceptors in vibrissa follicles from different species seem to have similar response properties, although some animals whisk and others do not.

Received Jan. 9, 2008; revised March 7, 2008; accepted April 6, 2008

This work was supported by European Community Grant IST-2000-28127 and the Volkswagen Foundation.

Correspondence should be addressed to Dr. Christiane Vahle-Hinz, Department of Neurophysiology and Pathophysiology, University Medical Center Hamburg-Eppendorf, Martinistrasse 52, 20246 Hamburg, Germany. E-mail: vahle-hinz@uke.de.

DOI:10.1523/JNEUROSCI.0089-08.2008

Copyright $\odot 2008$ Society for Neuroscience $\quad$ 0270-6474/08/285359-10\$15.00/0
The ascending transmission of signals appears to preserve precision of timing and high-frequency components of vibrissa movements in the principal trigeminal nucleus and the ventral posteromedial nucleus (VPM) of the thalamus (Vahle-Hinz and Gottschaldt, 1983; Deschênes et al., 2003; Vahle-Hinz et al., 2007). The picture seems completely different at the level of the barrel cortex [primary somatosensory cortex (S1)]. Here, studies in anesthetized rats have reported phase locking only up to $\sim 40$ $\mathrm{Hz}$ whisker vibration (Garabedian et al., 2003; Khatri et al., 2004; Kleinfeld et al., 2006), and coding strategies other than temporal patterning or phase locking have been put forward for highfrequency signals (Arabzadeh et al., 2003, 2004; Hartmann et al., 2003; Neimark et al., 2003; Andermann et al., 2004; Moore, 2004; Kleinfeld et al., 2006). Other studies have demonstrated oscillations at hundreds of hertz evoked by short pulse vibrissal stimuli in intracellular recordings and local field potentials (Jones and Barth, 1999; Jones et al., 2000; Barth, 2003), which indicate that the cortical network does possess the prerequisites for generating high-frequency activity. In awake rats, only relatively coarse stimuli, air puffs, or variable-width apertures have been used, which preclude an analysis of timing precision in the submillisecond range (Krupa et al., 2001; Melzer et al., 2006b).

Thus, the available data suggest an intriguing puzzle. Although high-frequency components of vibrissa movements, reflecting subtle surface irregularities, are precisely encoded by the peripheral mechanoreceptors and transmitted by the ascending pathway, there is no convincing evidence that these temporal 
patterns are used by the cortical circuitry. The data of the present study may contribute to resolving this issue. Using light carefully controlled isoflurane anesthesia, we show that $\mathrm{S} 1$ neurons of rats are readily able to phase lock to continuous highfrequency whisker vibrations above 100 Hz. In previous studies, such highfrequency phase locking may have been masked by suppressive effects of the anesthetics used (Detsch et al., 1999; Friedberg et al., 1999; Sceniak and MacIver, 2006; Vahle-Hinz et al., 2007).

\section{Materials and Methods}

Surgical preparation. This study was performed after approval of the Hamburg Administration of Health and Consumer Protection on 18 adult Wistar rats (400 g body weight) using methods reported previously in detail (Detsch et al., 1999; Vahle-Hinz et al., 2007). Anesthesia was induced by inhalation of isoflurane via a mask, a cannula was inserted into the trachea, and the lungs were mechanically ventilated with $1.5-2.0$ vol\% end-tidal isoflurane in $100 \%$ oxygen during the surgical preparation. Inspired and end-tidal isoflurane concentrations as well as end-tidal $\mathrm{CO}_{2}$ concentration were monitored continuously (Capnomac; Datex, Helsinki, Finland). The femoral vein was cannulated for administration of vecuronium bromide $\left(4 \mathrm{mg} \cdot \mathrm{kg}^{-1} \cdot \mathrm{h}^{-1}\right)$ for muscle relaxation. The heart rate was monitored continuously via an electrocardiogram, and the body temperature was maintained at $37.0 \pm 0.5^{\circ} \mathrm{C}$ by means of a feedbackcontrolled heating pad coupled to a rectal probe. A parieto-occipital electrocorticogram (ECoG) was displayed throughout the experiment to aid assessment of anesthetic depth in addition to tests of absence of cardiovascular responses when noxious pinching of a paw was applied. The external auditory meati were treated with lidocaine, and the animal's head was mounted in a stereotaxic holder with blunt ear bars in the flat skull position. A unilateral $3 \times 4 \mathrm{~mm}$ craniotomy was performed above the barrel cortex, the dura was removed, and the exposed cortical surface was covered with agar. At the end of the experiments, the animals were killed under $4.0 \mathrm{vol} \%$ isoflurane with potassium chloride intravenously, and the brain was removed and fixed by immersion in $4 \%$ glutaraldehyde in phosphate buffer. After dehydration, $50 \mu \mathrm{m}$ frozen sections were made and stained with $\mathrm{cr}-$ esyl violet for histological verification of the recording sites.

Neuronal recording and stimulation. Concentrations of isoflurane $(0.9-1.5 \mathrm{vol} \%)$ were adjusted during the recording session to the individual rat's susceptibility to the anesthetic as determined by the absence of pinch-induced movement, ECoG, and cardiovascular responses. Four individually movable glass-covered tungsten microelectrodes ( $1 \mathrm{M} \Omega$ impedance at $1 \mathrm{kHz}$ ) were used for simultaneous recordings of extracellular action potential (spike) discharges of multiunit and single-unit activities at four sites within the barrel cortex. The electrodes were inserted perpendicularly at 1-2 $\mathrm{mm}$ distances, and the principal whisker of the receptive fields was determined by manual movement of the whiskers while slowly lowering the electrodes. Most recordings were made at depths of $50-550 \mu \mathrm{m}$, corresponding to cortical layers $2 / 3$ and 4 , as measured from the cortical surface and verified histologically by small electrolytic lesions $(-4 \mu \mathrm{A}$ for $30 \mathrm{~s})$ applied through the tip of the recording electrode in some cases. The neuronal activity was amplified, filtered, displayed on a personal computer, and stored with $25-35 \mathrm{kHz}$ sampling rate on a hard disk using the Alpha-Map Data Acquisition System (Alpha Omega Engineering, Nazareth, Israel). Cortical neurons responding with sustained spike discharges to vibration of whiskers were selected. As sustained responses, we considered those in which spike discharges occur continuously during the entire duration of the stimulus and with a rate $>15$ spikes/s above that outside periods of stimulation (ongoing activity). A feedback-controlled electromechanical stimulator (Somedic, Stock-

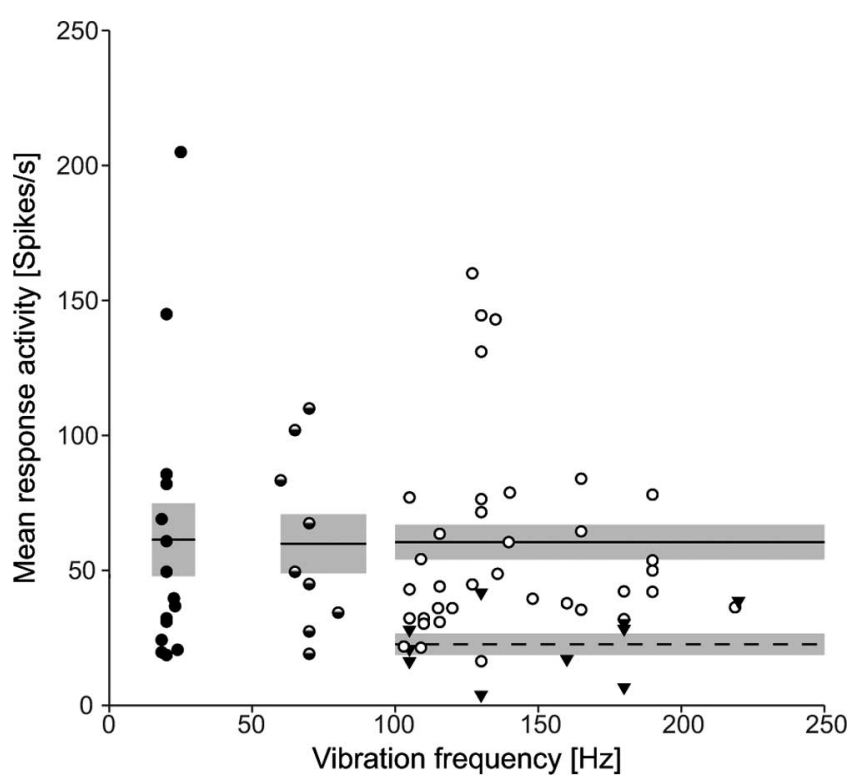

Figure 1. Mean firing rates of all responses classified as phase locked (circles; $n=60$ ) and of 10 single units (triangles) plotted as a function of the whisker vibration frequency. Horizontal lines and gray shades indicate the means \pm SEMs for the three groups (low-, medium-, and high-frequency responders: filled, half-filled, and open circles) and the single units (dashed line, triangles). Note that, for the three groups, data were entered only of the highest response rate from each recording site.

holm, Sweden) was used to produce whisker movement with constant displacement amplitudes. Single whiskers were attached to the stimulator probe $\sim 5 \mathrm{~mm}$ from the skin and were moved by controlled sinusoidal-shaped stimuli $(10-700 \mathrm{~Hz}, 20-600 \mu \mathrm{m}$ amplitude, 0.5 or $1 \mathrm{~s}$ duration delivered at $5 \mathrm{~s}$ intervals) in rostrocaudal direction. For each unit, the principal whisker was chosen and the stimulus parameters (amplitude and frequency of vibration) were adjusted, allowing for optimal and robust responses. To allow pooling of the data, three ranges of vibratory frequencies were used, i.e., high $(>100 \mathrm{~Hz})$, medium $(60-90 \mathrm{~Hz})$, and low $(15-30 \mathrm{~Hz})$ frequencies.

Data analysis. Off-line analyses were performed with several programs for single-unit sorting (Offline-Sorter; Plexon, Dallas, TX) to generate time stamps, peristimulus time histograms (PSTHs), and phase histograms (Spike2 software; Cambridge Electronic Design, Cambridge, UK), as well as time-frequency analyses and statistics (custom-written Matlab routines). Fifty consecutive responses to each kind of stimulus were used in all analyses. PSTHs (bin width $1 \mathrm{~ms}$ ) were generated from the neuronal responses, and the response activity per stimulus was determined as mean discharge frequency (spikes per second) above ongoing activity, 

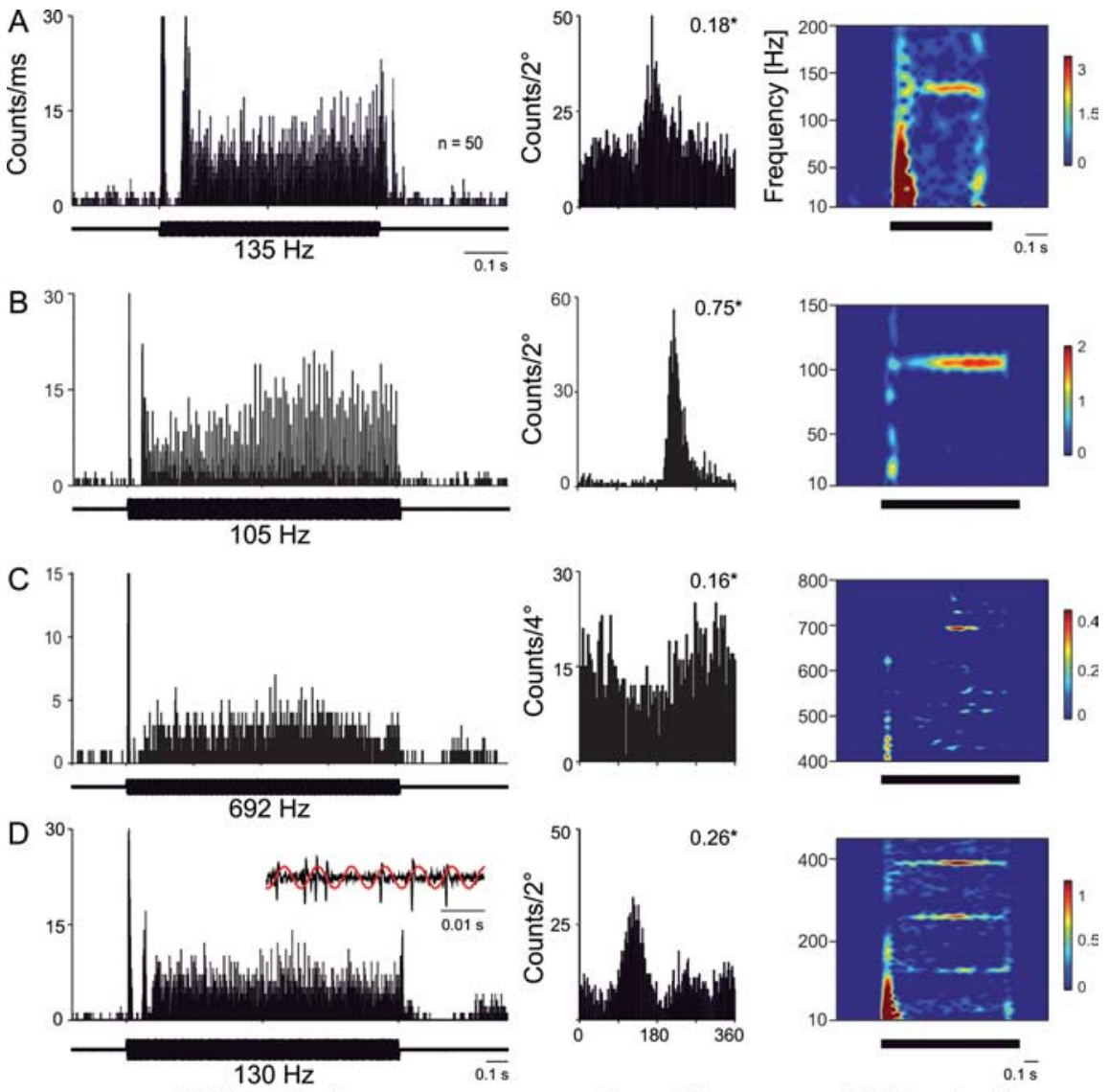

Whisker vibration

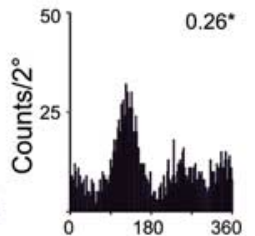

Phase $\left[{ }^{\circ}\right]$
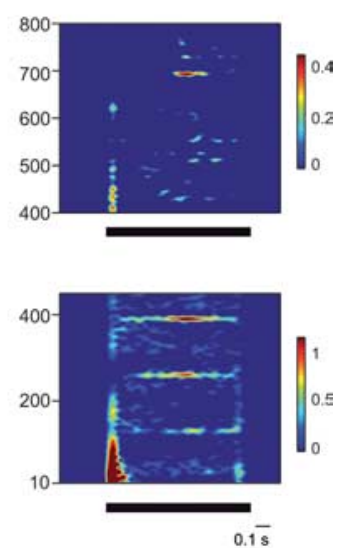

Whisker vibration

Figure 2. Responses of four $S 1$ multiunits to high-frequency $(>100 \mathrm{~Hz})$ vibration of their principal whiskers for $0.5 \mathrm{~s}(\boldsymbol{A})$ and 1s (B-D). Left column, PSTHs (bin width $1 \mathrm{~ms}$ ) of the responses to 50 consecutive stimuli (bottom traces). Middle column, Phase histograms generated from these responses show precision of phase locking (numbers in the top right corner indicate the vector strengths; ${ }^{*} p<0.01$ ). Right column, Time-frequency plots of the stimulus-locked activity showing the increase of evoked power during stimulation (bar at the bottom). The color scale indicates the power increase in arbitrary units. A band of increased power representing the stimulation frequency is present in all cases. In addition, the time-frequency plot in $\boldsymbol{D}$ exhibits bands at two higher harmonics ( 260 and $390 \mathrm{~Hz}$ ), reflecting spikes that give rise to additional peaks in the phase histogram (middle). The occurrence of such phase-shifted additional spikes is also clearly visible in epochs from single trials, as shown in the inset in the PSTH (left).

calculated from the discharge rates measured during stimulus application and the $200 \mathrm{~ms}$ period preceding stimulus onset, respectively. The units were assigned to a group (high-, medium-, and low-frequency responders) according to their best responses. Data from units responding equally well to stimuli in two or three frequency ranges were assigned to these different response groups.

Phase histograms were generated by plotting the spikes against the phase of the vibratory stimulus cycle. For this analysis, the ON responses were excluded, and only parts of the responses beyond $200 \mathrm{~ms}$ after stimulus onset were used. Thereby, $300-28,000$ cycles were analyzed for each set of 50 responses, depending on the stimulus frequency and duration. The temporal consistency of spike timing across stimulus cycles was analyzed by using vector strength (VS) as a measure. VS is calculated by fast Fourier transformation (FFT) and quantifies the degree of coupling of the spikes to the same phase of a vibratory cycle during each repetitive cycle of stimulation (Goldberg and Brown, 1969; Khatri et al., 2004). A VS value of 1 indicates perfect phase locking to the stimulus, whereas a VS of 0 indicates absence of a consistent phase relationship of the spikes to the stimulus. Statistical significance was tested according to the method used by Durand and Greenwood (1958), and a $p<0.01$ was considered significant to classify phase-locked (PLR) and non-phase-locked (NLR) responses. The phase in itself at which the spikes occurred was not analyzed, because elicitation of spikes in the mechanoreceptor axon depends on the position of the receptor alongside the hair shaft in the follicle with respect to the propagation of the wave along the whisker, which in turn depends on the position of the stimulator probe and the frequency and amplitude of the vibration.

A frequency-domain analysis of the spike responses was obtained by applying FFT (160 ms window and $157 \mathrm{~ms}$ overlap) to the time stamps of the spikes transformed into continuous data with $3 \mathrm{kHz}$ sampling rate. Time-frequency plots showing absolute power of the frequency components were obtained by applying FFT analysis to the mean of 50 consecutive responses, resulting in a measure of the evoked activity, which is by definition strictly locked to stimulus onset. Oscillatory activity that occurs with a jitter in onset latency or phase from trial to trial is lost during the averaging process. This activity, however, is recovered when the FFT analysis is applied to each single response before averaging, reflecting the total activity that comprises both stimulus-locked (evoked) and non-locked (induced) activities (Tallon-Baudry and Bertrand, 1999). To obtain only the non-locked activity, first the mean evoked response was subtracted from each single response, and then the FFT analysis was applied to each single response, 50 of which were then averaged. Comparisons of different responses and stimulation groups were made with Wilcoxon's tests. A $p<0.01$ was considered significant. Data are given as mean \pm SEM.

\section{Results}

Quantitative data were derived from 57 multiunits of the S1 activated by vibratory stimulation of single whiskers at frequencies of 15-700 Hz. Data from 10 single units sorted from these multiunits or recorded individually were analyzed separately and are reported below. Seventeen multiunits were activated by vibration at several different frequencies; thus, a total number of 79 different vibratory responses were used for the analyses. The mean discharge rate of all vibratory responses $(n=$ 79 ) increased to $55.5 \pm 4.2$ spikes/s above ongoing activity during the sustained discharges (response activity) and was $14.6 \pm 2.0$ spikes/s outside periods of stimulation (ongoing activity). Sixty responses were classified as PLR and had a mean rate of $1.1 \pm 0.2$ spikes per cycle, whereas the 19 NLRs had a significantly lower rate $(0.4 \pm 0.1$ spikes per cycle, $p<0.005$; Table 1$)$. The mean discharge rates of PLRs did not differ for low-, medium-, and high-frequency stimulation (Fig. 1), as well as for NLRs during medium-frequency stimulation, but were lower for NLRs during high-frequency stimulation. The associated ongoing activities were significantly higher for NLRs than for PLRs.

\section{Responses to high-frequency vibration}

Responses to high vibratory frequencies $(>100 \mathrm{~Hz})$ occurred phase locked to the vibratory cycles (VS of $0.25 \pm 0.03$ ) in 36 PLRs (72\%), with a discharge rate per cycle approximately twice as high $(0.45 \pm 0.05$ spikes per cycle) than in the 14 NLRs $(28 \%$; $0.24 \pm 0.03$ spikes per cycle; VS of $0.04 \pm 0.01)$. For 30 of the PLRs, time-frequency analysis revealed an increase of evoked power at the stimulation frequency. For six PLRs, bands of evoked power were not clearly discernible, presumably because 
spikes occurred phase locked only intermittently but not continuously to several consecutive cycles of vibratory stimulation.

Examples of PLRs are shown for four multiunits in Figure $2 A-C$. A typical response (Fig. 2A, $B$, PSTHs in left column) consisted of a marked ON burst, followed by a discharge pause of 50-100 ms, presumably of inhibitory origin as evidenced by the subsequent rebound burst. Then, a regular discharge followed that was entrained by the vibration frequency as substantiated by the peak in the phase histograms (Fig. $2 A, B$, middle column) and the band of increased evoked power in the time-frequency plots (right column). In $39 \%$ (14 of 36) of the PLRs, this entrainment increased with stimulus duration (see also Figs. 4, 6) and in 36\% (13 of 36), it remained constant, whereas in 25\% (9 of 36) of PLRs, a decrease of entrainment and spike rate indicated response adaptation. The highest frequency, to which a unit responded in a phase-locked manner, was $692 \mathrm{~Hz}$ (Fig. 2C). The mean response rate in this case was 30 spikes/s, and the phase histogram shows that the spikes were elicited significantly more often by one movement direction; thus, precision of spike elicitation amounted to $<1 \mathrm{~ms}$. A band representing stimulus frequency is discernible in the middle part of the stimulus epoch in the time-frequency plot (Fig. 2C). In the case of the recording shown in Figure $2 D$, some cycles of the $130 \mathrm{~Hz}$ vibration elicited three spikes (see original record in inset). This three-to-one response component occurred consistently as reflected by the three significant peaks in the phase histogram and three bands of evoked power at 130,260, and $390 \mathrm{~Hz}$ in the time-frequency plot.

Examples of four single units are shown in Figures 3 and 4 . The single unit of Figure 3 was activated best by vibration of the whisker at $220 \mathrm{~Hz}$. The PSTH reflects the sustained response, but only the phase histogram reveals the phase locking of the spike discharges to the vibratory cycles, and the time-frequency plot shows the 1:1 discharges over several consecutive cycles by the emergence of a band at $220 \mathrm{~Hz}$ (Fig. $3 a-c$ ). The original record of this unit shows the sustained responses elicited by four consecutive stimuli and its low ongoing activity (Fig. $3 d$ ). The variability of the individual responses is reflected in Figure $3, e, h$, and $i$, where response entrainment increases $(e, i)$ or decreases $(h)$ during the stimulus epoch. At expanded timescale (Fig. $3 f$ ), the coupling of four of six spikes to the half-phase of the downward direction is visible. Thus, the precision of spike generation amounts to less than a half-cycle, i.e., $<2.3 \mathrm{~ms}$. Figure $4 A-C$ depicts data from three single units sorted from three multiunit recordings. The ON responses are missing from the PSTHs because individual spike shapes cannot be identified within the bursts of action potentials elicited by all units at stimulus onset. In the case of the single unit shown in Figure $4 C$, the pronounced

g b
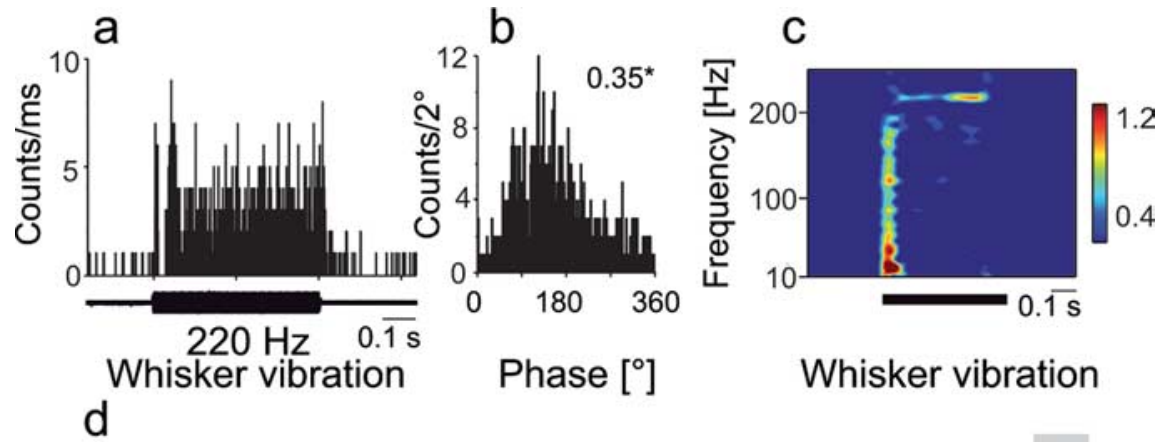

Whisker vibration

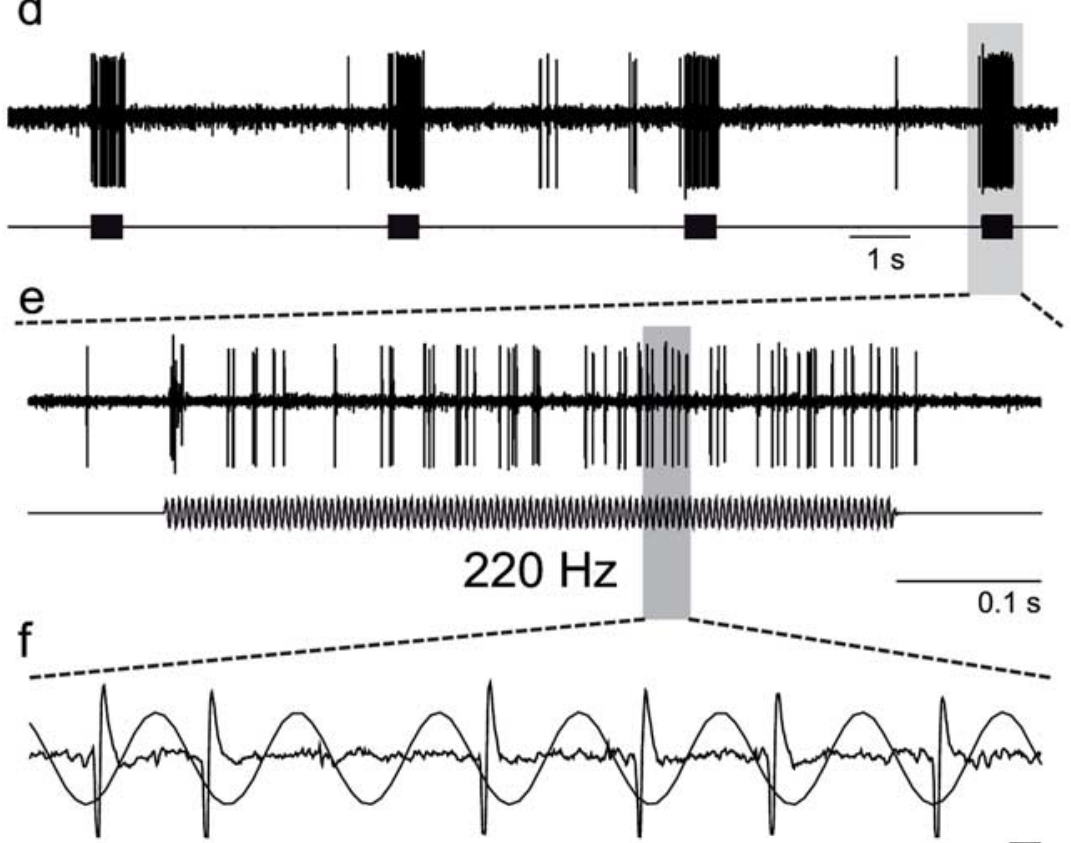

h

$1 \overline{\mathrm{ms}}$

Figure 3. Single-unit responses to $220 \mathrm{~Hz}$ vibration of the principal whisker. $\boldsymbol{a}-\boldsymbol{c}$, PSTH (a), phase histogram (b), and time-frequency plot of 50 consecutive responses (c) with the same conventions as in Figure 2 . $\boldsymbol{d}-\boldsymbol{f}$, The raw spike trace (d) shows four consecutive responses, the last of which is displayed at expanded timescales below $(\boldsymbol{e}, \boldsymbol{f})$. Although there is no continuous $1: 1$ response, the majority of spikes occur locked to a consistent phase of the movement cycle. $\boldsymbol{g}$, Spike waveforms of the single unit. $\boldsymbol{h}, \boldsymbol{i}$, Additional single response epochs, highlighting the variability of the response pattern.

discharge pause after stimulus onset is missing, and the spikes occur phase locked to each vibratory cycle (see inset). The overall response pattern of the sustained discharges is similar to that of the multiunits showing an increase in vibratory entrainment during later parts of the stimulus epoch in two units (Fig. $4 A, B$, PSTHs, left column, and time-frequency plots, right column), whereas the response strength is greatest during the early part of the stimulus epoch in one unit $(C)$. Phase locking appears to be more precise than for multiunits, as reflected by the sharp peak in the phase histograms (Fig. 4, middle column) and a VS of $>0.8$. The mean response rate of all 10 single units increased to $22.6 \pm$ $4.0 \mathrm{spikes} / \mathrm{s}$ above ongoing activity $(1.7 \pm 0.8 \mathrm{spikes} / \mathrm{s})$, and the discharge rate per cycle was $0.16 \pm 0.03$ with a VS of $0.47 \pm 0.10$. Comparison with the data from the multiunit recordings suggests that approximately three single units may have contributed to each of the multiunit recordings. 

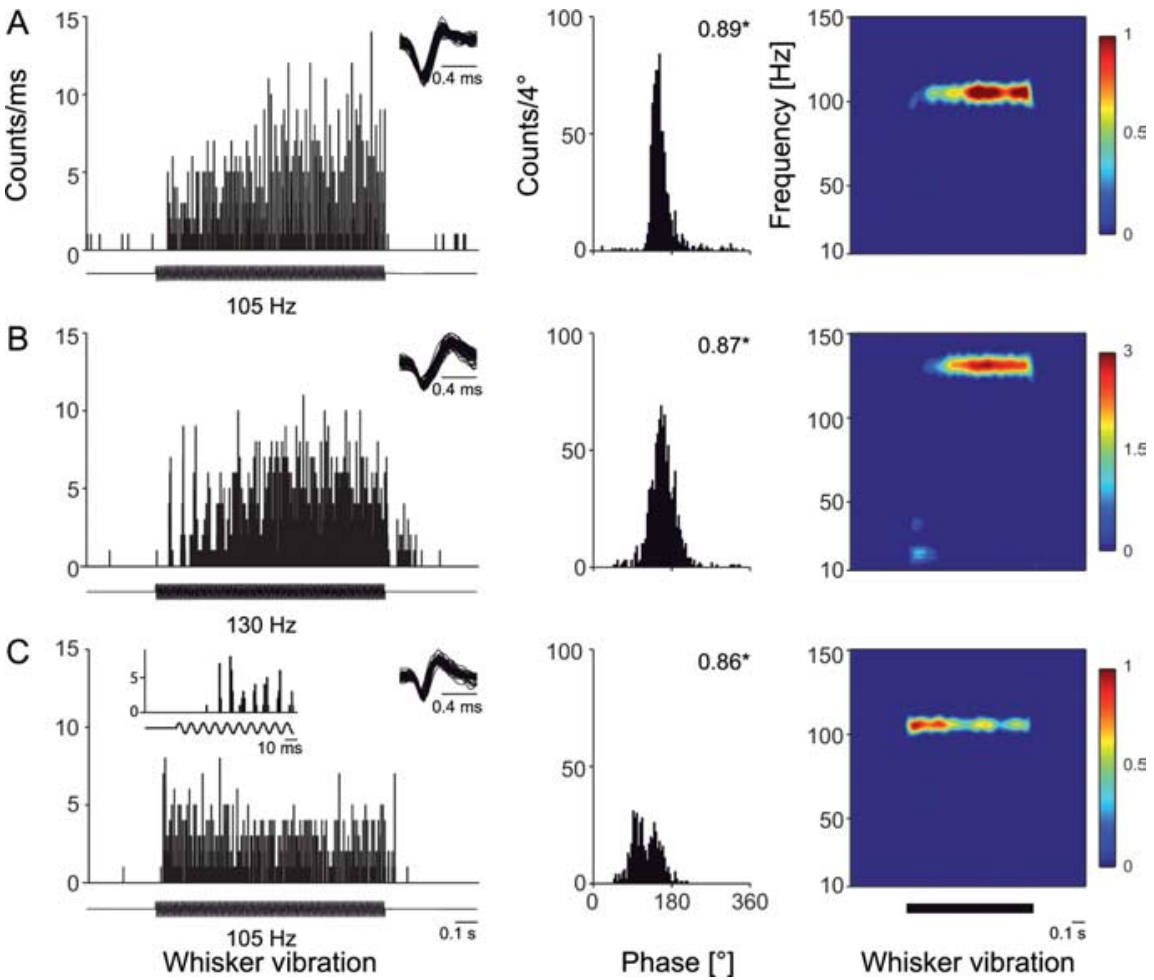

Figure 4. $\quad \boldsymbol{A}-\boldsymbol{C}$, Responses of three $\mathrm{S} 1$ single-units $(\boldsymbol{A}-\boldsymbol{C})$ sorted from three multiunit recordings to high-frequency $(>100 \mathrm{~Hz})$ vibration of their principal whiskers for $1 \mathrm{~s}$. Left, middle, and right columns, PSTHs, phase histograms, and time-frequency plots, respectively, with the same conventions as in Figure 2. Insets above the PSTHs show the spike waveforms, and the inset in C shows initial part of PSTH at expanded timescale. The response in $\boldsymbol{B}$ has been derived from 30, and those in $\boldsymbol{A}$ and $\boldsymbol{C}$ from 50 consecutive stimuli. $A$ band of increased power representing stimulation frequency is present in the time-frequency plots in all three cases.
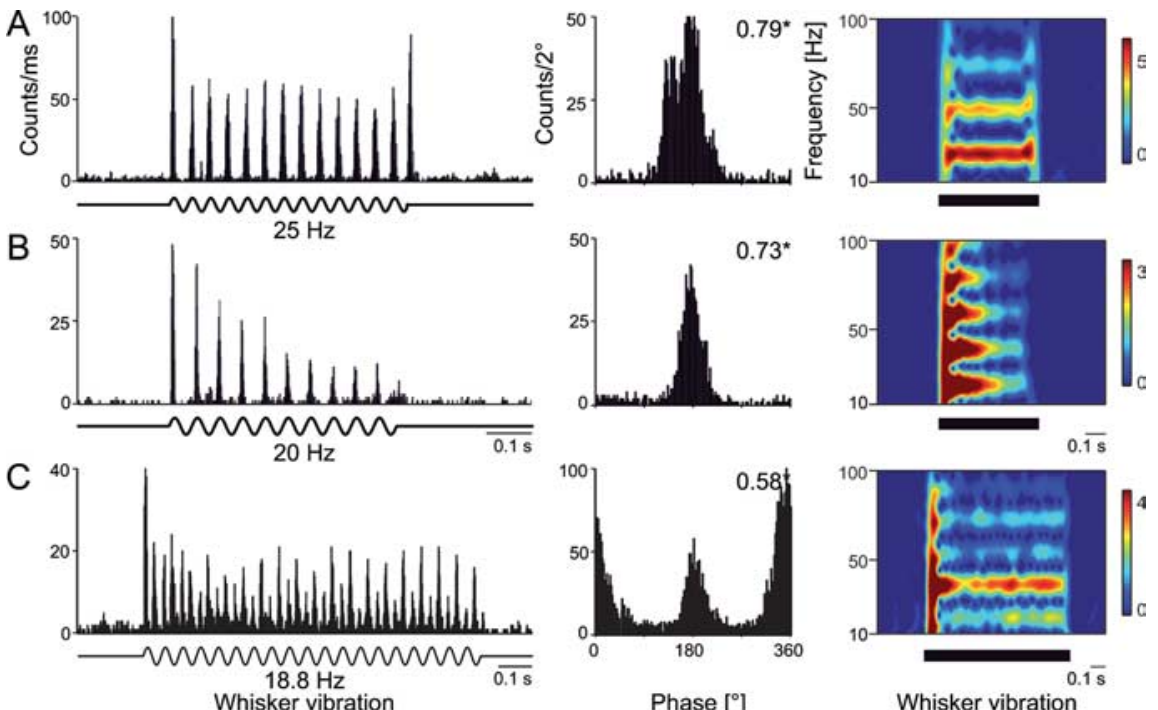

Figure 5. $\quad \boldsymbol{A}-\boldsymbol{C}$, Responses of three cortical multiunits to low-frequency vibration of their principal whiskers for $0.5 \mathrm{~s}(\boldsymbol{A}, \boldsymbol{B})$ and $1 \mathrm{~s}(\boldsymbol{C})$. Left, middle, and right columns, PSTHs, phase histograms, and time-frequency plots, respectively, with the same conventions as in Figure 2. The PSTHs show that the spikes were elicited phase locked to the cycles of the sinusoidal vibrations (bottom traces). The units in Cresponded phase locked to both movement directions. The time-frequency plots show that the $0 \mathrm{~N}$ responses consist of a wide band of frequencies, and bands at the stimulus frequencies as well as higher harmonics occur during the stimulation epoch. The main increase in power occurs at the next higher harmonic of the stimulation frequency in $\boldsymbol{C}$.

approximately twice as high $(3.0 \pm 0.6$ spikes per cycle) in the PLRs compared with the NLR (1.6 spikes per cycle).

Low-frequency stimulation elicited multiple spikes per cycle that occurred locked to a specific phase of the whisker movement, resulting in one peak per cycle in the PSTH and the phase histogram (significant VS, ${ }^{\star} p<0.01$ ) in the multiunits shown in Figure 5, $A$ and $B$, thereby representing directionally sensitive responses. The time-frequency plots show a band of evoked power representing stimulus frequency and also evidence for higher harmonics. Although the responses of the unit shown in Figure $5 A$ occur at constant strength throughout the stimulus epoch, a marked adaptation is present in the responses of the unit shown in Figure $5 B$. A $1: 1$ coding of low-frequency vibrations was present in $71 \%$ (10 of 14) of the PLRs. In $29 \%$ ( 4 of 14) of the PLRs, both movement directions elicited spikes, again locked to a specific phase of the cycle. This response pattern is evident in the PSTH and the phase histogram of the example in Figure $5 C$. Both peaks in the phase histogram are significant, thus resulting in a 2:1 vibratory response that is reflected in the prominent band at $37.6 \mathrm{~Hz}$ in the time-frequency plot. Bands at the fundamental stimulus frequency or higher harmonics occurred in 93\% (13 of 14) of the PLRs. When the units discharged in response to both movement directions, the highest power always was present in the band of the second harmonic.

Responses to medium vibratory frequencies $(60-90 \mathrm{~Hz})$ occurred phase locked to the vibratory cycles (VS of $0.33 \pm$ 0.22 ) in 9 PLRs (69\%), with a discharge rate per cycle $(0.94 \pm 0.2$ spikes per cycle) $\sim 20 \%$ higher than in the four NLRs $(0.76 \pm 0.60$ spikes per cycle; VS of $0.04 \pm$ $0.01)$. An increase of evoked power at the stimulus frequency was evident in the time-frequency plots of all PLRs. An example is shown in Figure 6, with phase-locked responses of a multiunit to 60 and $90 \mathrm{~Hz}$ whisker vibration. The entrainment to the vibration increased during the $1 \mathrm{~s}$ epoch of stimulation (see PSTHs and time-frequency plots), and the peak in the phase histograms shows the precise coupling to a specific phase of the vibratory cycles.

\section{Wide-band vibratory responses}

In the majority of cases in our dataset, only the best frequency was studied to which the

Responses to low- and medium-frequency vibration Responses to low vibratory frequencies $(15-30 \mathrm{~Hz})$ occurred phase locked to the vibratory cycles in all (14 PLRs; VS of $0.42 \pm 0.06$ ) but one unit tested (NLR; VS of 0.01). The discharge rate per cycle was units responded. For some recording sites, it was noted, however, that units responded to a broad range of frequencies. These were studied in detail in five cases, an example of which is shown in Figure 6 with responses of one multiunit to whisker vibration at 
frequencies between 20 and $560 \mathrm{~Hz}$. The response rates were above 35 spikes/s for all frequencies, and significant phase locking was observed over a broad range of frequencies between 20 and $320 \mathrm{~Hz}$ and also at $460 \mathrm{~Hz}$ (Fig. 6, phase histograms, middle column). In case of the highest frequencies, the precision of spike timing was better than $1 \mathrm{~ms}$. The vector strength, however, was negatively correlated with frequency. Bands of evoked power representing the stimulus frequency were prominent in the time-frequency plots between $20-320 \mathrm{~Hz}$ vibration (Fig. 6, right column). In three other units with wide-band vibratory responses, phase locking was present at all frequencies tested (10-200, 20-135, and $20-165 \mathrm{~Hz}$, respectively). In the remaining unit, phase locking occurred only in the low and medium frequency ranges (20 and $65 \mathrm{~Hz}$ but not at $158 \mathrm{~Hz}$ ).

In another eight cases, stimulation was performed at low and high frequencies but not in the medium frequency range. In all of these cases, signals were phase locked at low frequencies and, in six of eight cases, also at high frequencies. The highest vibration frequency that elicited 1:1 responses in the barrel cortex under isoflurane anesthesia was $320 \mathrm{~Hz}$.

\section{Gamma-band activity}

Entrainment of the spike discharges with the vibratory stimuli occurring continuously or even increasing during the stimulation epoch was observed for responses to high-frequency vibrations more often (75\% of PLRs) than adaptation (25\%). The occurrence of oscillatory response components not strictly locked to vibratory whisker movements was studied further by time-frequency analyses of the total and induced activities (Fig. 7). Because the phase and latency of induced oscillatory activity varies across stimulus presentations, this fraction of the response is cancelled out by the averaging process used to determine the evoked activity. Therefore, the timefrequency power was computed first for each single trial, 50 of which were then averaged (total activity) or which were averaged after subtraction of the evoked activity (induced activity). Multiunit responses to low- and medium-frequency vibration showed bands of evoked power at the stimulation frequency and its harmonics but no indication of additional induced activity (Fig. $7 A, B$ ). In contrast, prominent induced oscillations in the gamma-band frequency range were visible in the plots of total and induced activities for multiunits responding to high-frequency vibration (Fig. 7C,D). Interestingly, this induced gamma-band response emerges concomitantly with the spike timing entrainment, either during the later part of the stimulus epoch (see band of evoked $190 \mathrm{~Hz}$ in Fig. 7C) or shortly after stimulus onset (see band at $130 \mathrm{~Hz}$ evoked activity in Fig. 7D). $20-320 \mathrm{~Hz}$.
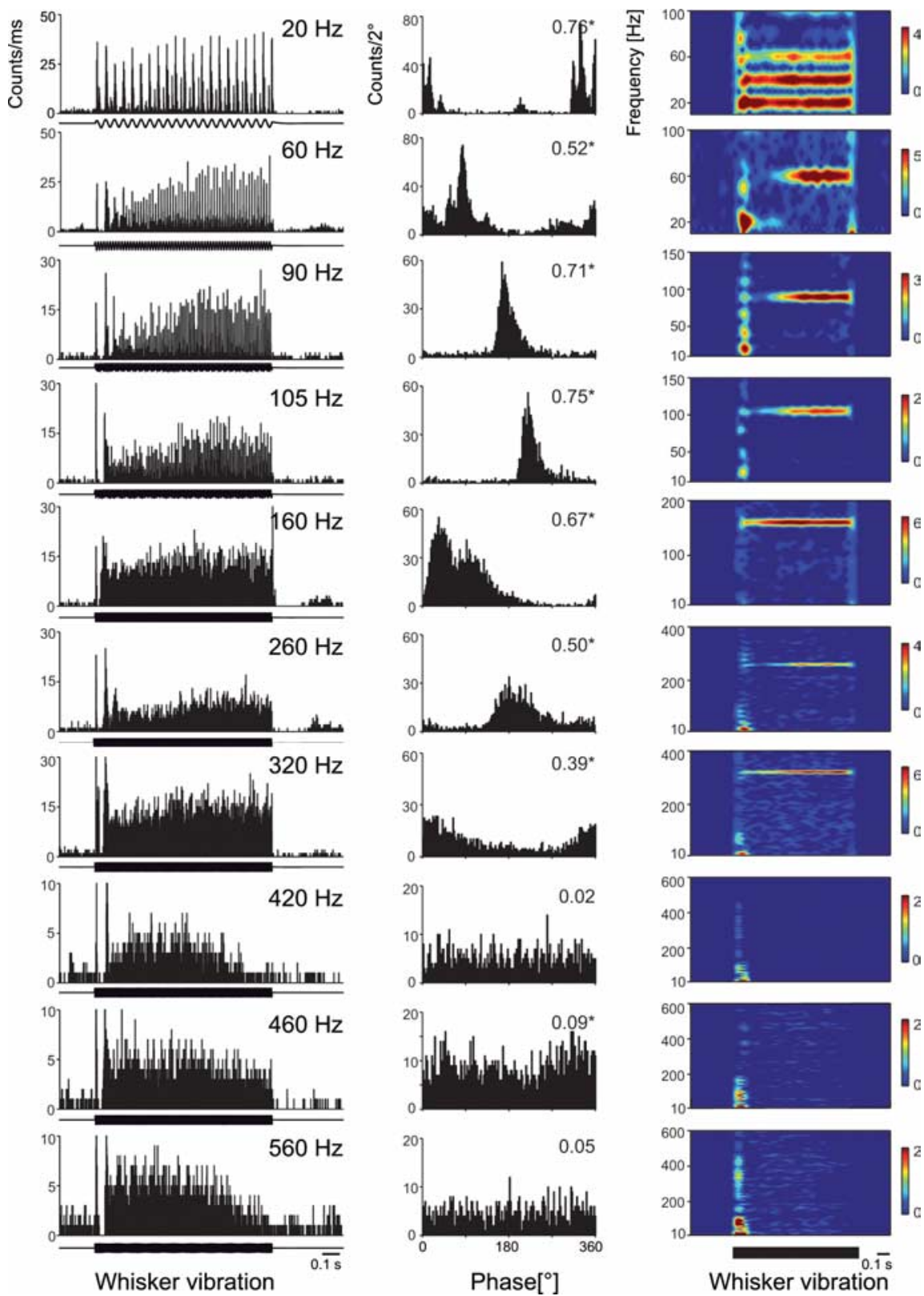

Phase $\left[{ }^{\circ}\right]$
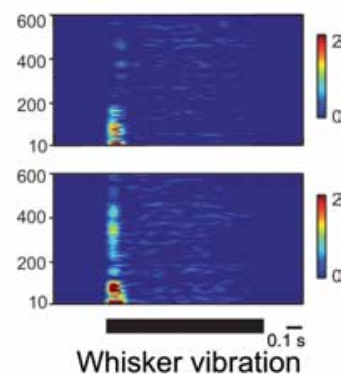

Figure 6. Wide-band responses of a multiunit to vibration of the principal whisker at $20-560 \mathrm{~Hz}$ (same unit as shown in Fig. $2 B$ ). Left, middle, and right column, PSTHs, phase histograms, and time-frequency plots, respectively, with the same conventions as in Figure 2. The sustained responses ( $>35$ spikes/s above ongoing activity) are phase locked to vibratory frequencies from 20 to $320 \mathrm{~Hz}$ and at $460 \mathrm{~Hz}$, with bands of evoked power occurring in the time-frequency plots for vibratory frequencies of

When the induced activity was analyzed for the population average of 19 PLRs, a clear increase in power in the gamma band $(30-70 \mathrm{~Hz})$ was observed during the stimulus epoch (Fig. 8A). To compare the changes in frequency components during stimulation with those during prestimulus ongoing activity, relative power was computed (Fig. $8 \mathrm{~B}$ ). A $1 / f$ frequency distribution was present during ongoing activity, but a prominent shift to gammaband frequencies occurred especially during the second half of the stimulus epoch. In the population average, this induced gamma-band response reached significance only for the second half of the responses (Fig. 8C). Moreover, a significant decrease of non-phase-locked oscillatory activity was observed at lower frequencies, $\sim 20 \mathrm{~Hz}$ (Fig. 8C). 


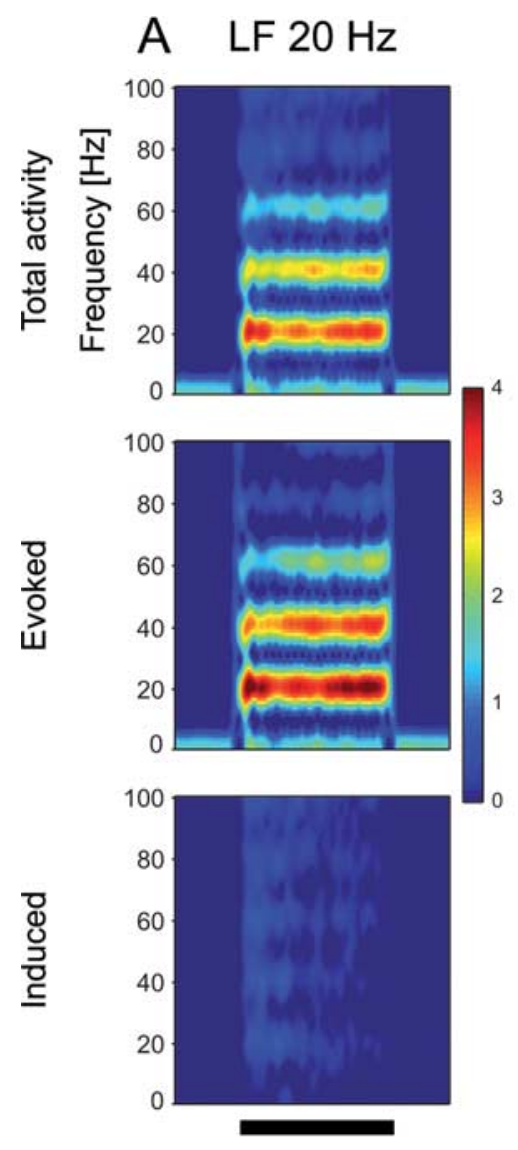

B $\quad$ MF $60 \mathrm{~Hz}$
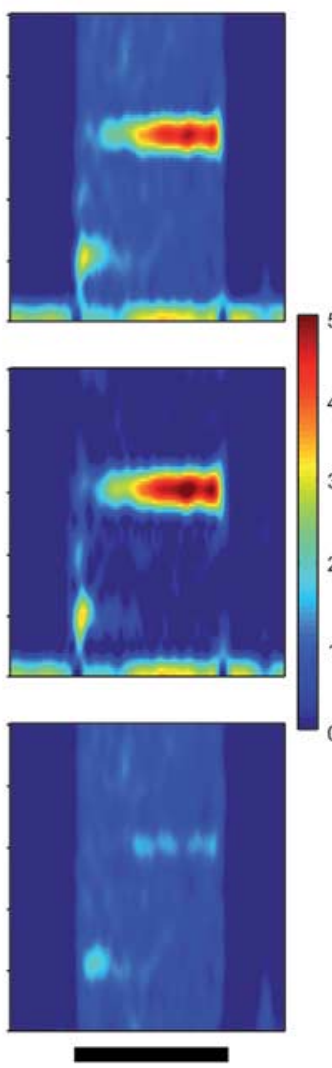

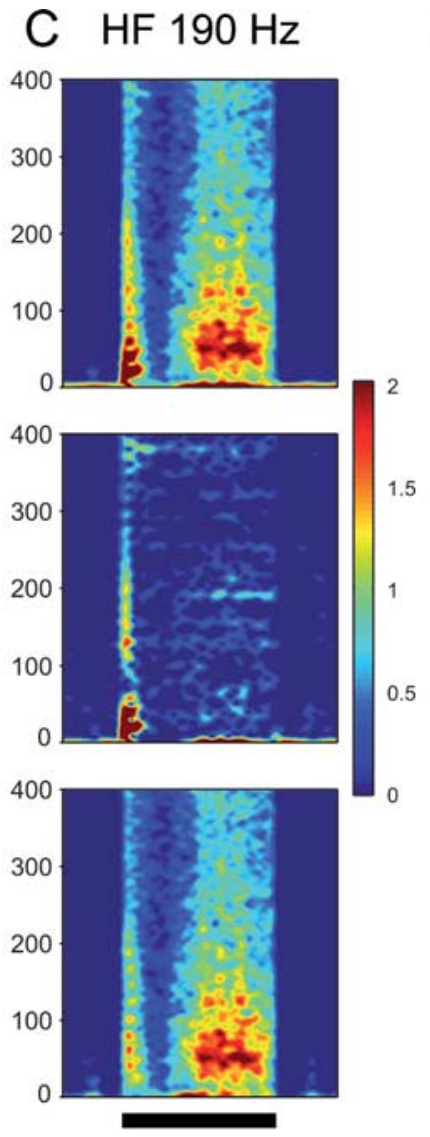

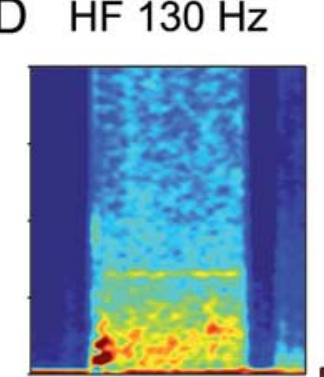

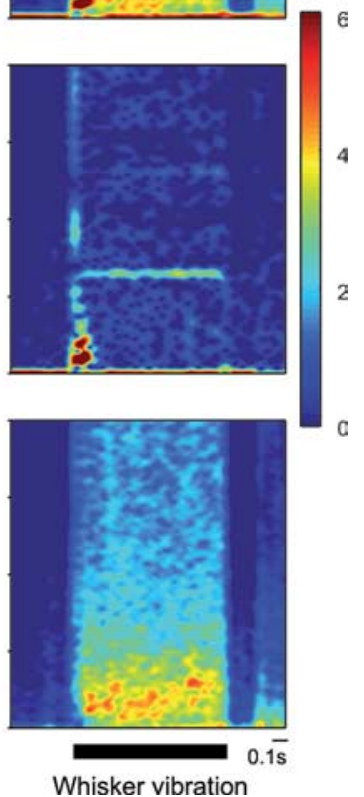

Figure 7. Time-frequency analysis of total (top row), evoked (middle row), and induced (bottom row) power of cortical responses to whisker vibration. $\boldsymbol{A}-\boldsymbol{D}$, Four different examples are given representing the low-frequency (LF), medium-frequency (MF), and high-frequency (HF) stimulation groups. Bands of increased power at the stimulation frequency are visible in the evoked and total activities of each case $(\boldsymbol{A}-\boldsymbol{D})$ as well as harmonics during low-frequency stimulation $(\boldsymbol{A})$. Additional induced activity only emerges during high-frequency stimulation $(\boldsymbol{C}, \boldsymbol{D})$. Black bars at bottom mark the stimulation periods. The color scale indicates the power increase in arbitrary units.

\section{Discussion}

This is the first report about spike discharges of S1 neurons entrained by high-frequency vibrations of a whisker in a phaselocked manner for long stimulus durations. In our dataset, phase-locked responses occurred up to vibratory frequencies close to $700 \mathrm{~Hz}$, and 1:1 responses were present up to $\sim 300 \mathrm{~Hz}$. Also, the discharge rates elicited by whisker vibration were higher than reported before for multiunits with a mean of $60.5 \pm 5.2$ spikes/s, which occurred continuously for several hundred milliseconds in our sample of 60 PLRs. Adaptation of the responses was rarely seen; rather, entrainment to the vibratory frequency remained constant or increased with stimulus duration. Another significant finding of the present study is that phase locking of the spikes can be accompanied by the emergence of induced (nonphase locked) activity in the gamma-band range. Our finding of high-frequency responses hitherto unobserved in the barrel cortex suggests the possibility that awake animals make use of the information about vibratory signals also at frequencies at hundreds of hertz, which are provided by the vibrissal mechanoreceptors.

The main difference between our study and previous measurements in anesthetized rats lies in the type of anesthesia. In most studies (Arabzadeh et al., 2003, 2004; Andermann et al., 2004; Melzer et al., 2006a), urethane anesthesia has been applied intraperitoneally, resulting in a relatively uncontrolled state of anesthesia over the course of the experiment. Urethane is widely used because a single intraperitoneal injection provides immobil- ity for surgical procedures and electrophysiological recordings for several hours. It is generally thought to only minimally affect circulation and respiration as well as neuronal activity at all stages of the CNS. The mechanisms of action of urethane have only begun to be elucidated recently. An in vitro study of recombinant neurotransmitter receptors in frog oocytes showed that urethane effected small but discernible changes in multiple receptor systems, including $\mathrm{GABA}_{\mathrm{A}}$, glycine, NMDA, AMPA, and acetylcholine receptors (Hara and Harris, 2002). A study using slices from rat visual cortex (Sceniak and MacIver, 2006) has recently reported a depression of neuronal spike output attributable to augmentation of a potassium leak conductance. In addition, the effects of urethane on subcortical stages may add to the suppressive effects on signal processing (Friedberg et al., 1999). Comparing three stages of urethane anesthesia by incremental intraperitoneal doses, Erchova et al. (2002) reported bursting activity that became more prominent and increasingly synchronized between barrels with increasing depth of anesthesia. Cortical responses to whisker stimulation were found only during bursts. Very similar observations are made during the burst-suppression mode induced by high concentrations of isoflurane (Detsch et al., 2002). In this respect, isoflurane and urethane anesthesia may be comparable. However, it appears that, at a level of anesthesia adequate for electrophysiological studies, isoflurane induces far less suppression of cortical activity than urethane. This is evidenced by (1) the absence of bursting in ongoing activity under isoflurane as 
is also seen in awake animals regardless of the well known stimulus-related oscillatory activity (Jones and Barth, 1999; Barth, 2003), (2) a generally higher neuronal activity as reflected in the higher rate of ongoing spike activity (Erchova et al., 2002), and (3) absence of adaptation to long-lasting stimulation (Andermann et al., 2004). Low rates of ongoing and evoked activities as well as prominent response adaptation within a few tens of milliseconds were also reported in studies using other anesthetics, such as pentobarbitone, ketamine-xylazine, halothane, or fentanyl (Deschênes et al., 2003; Garabedian et al., 2003; Khatri et al., 2004). Our data clearly suggest that high-frequency components of stimuli can be faithfully encoded by S1 neuronal discharges and that the absence of fast response components reported in the previous studies (for review, see Moore, 2004; Kleinfeld et al. 2006) is primarily attributable to the usage of anesthetics that block the emergence of fast rhythms in the thalamocortical system.

Despite the more faithful transmission of fast signals to the cortical level, the function of the thalamocortical circuits under light isoflurane still differs in important respects from processing in the awake state. A particularly important difference concerns the initial part of the S1 response. Only rarely (Fig. 4C) did we observe a vibratory response present immediately after stimulus onset. The majority of responses began with an ON burst, followed by a discharge pause and a subsequent rebound burst before the discharges became entrained to the stimulus vibration. This initial non-stimulus-entrained part lasted for 50-100 ms, which is longer than an awake behaving rat would wait for the relevant information. When a surface structure is relevant for a decision, rats usually attain this decision very fast after contact with the stimulus (Harvey et al., 2001; Krupa et al., 2001). The ON burst transmitted by the somatosensory system triggers intracortical feedback inhibition, which under most anesthetics is potentiated and thus more powerful than during wakefulness (Detsch et al., 1999, 2002). The same response pattern is present in VPM neurons under isoflurane anesthesia and can be suspended by blockade of $\mathrm{GABA}_{\mathrm{A}}$ receptors, the sites of action of isoflurane in the thalamus, thereby reestablishing sustained vibratory responses from stimulus onset (Vahle-Hinz et al., 2001, 2007). It is very likely that, in the awake animal, both thalamic input as well as cortical activity faithfully convey the continuous vibratory structure of the input from stimulus onset.

In addition to evoked high-frequency responses, our data provide evidence for intrinsically generated gamma-band oscillations, which correspond to temporal patterns that are not phase locked to stimulus transients. These induced oscillations in the range of $30-70 \mathrm{~Hz}$ only occurred with fast vibratory stimuli, accompanying the emergence of phase locking at the higher frequencies. With low- or medium-frequency vibration, in contrast, phase-locked components seemed to dominate this frequency range, possibly masking intrinsically generated gamma-band oscillations. Our observations complement a number of previous studies that have reported similar oscillatory activity in the somatosensory system. Work in slices from rodent somatosensory cortex has shown that the local circuitry is capable of generating rhythmic activity at gamma-band frequencies, based on an interplay of intrinsically bursting excitatory neurons, fast-spiking inhibitory interneurons, and modulatory transmitter systems (Chagnac-Amitai and Connors, 1989; Buhl et al., 1998). Studies on awake rats have demonstrated the occurrence of gamma oscillations in barrel cortex in relation to whisker stimulation (Jones and Barth, 1997) and tactile exploratory behavior (Hamada et al., 1999). Related evidence for fast oscillatory activ-

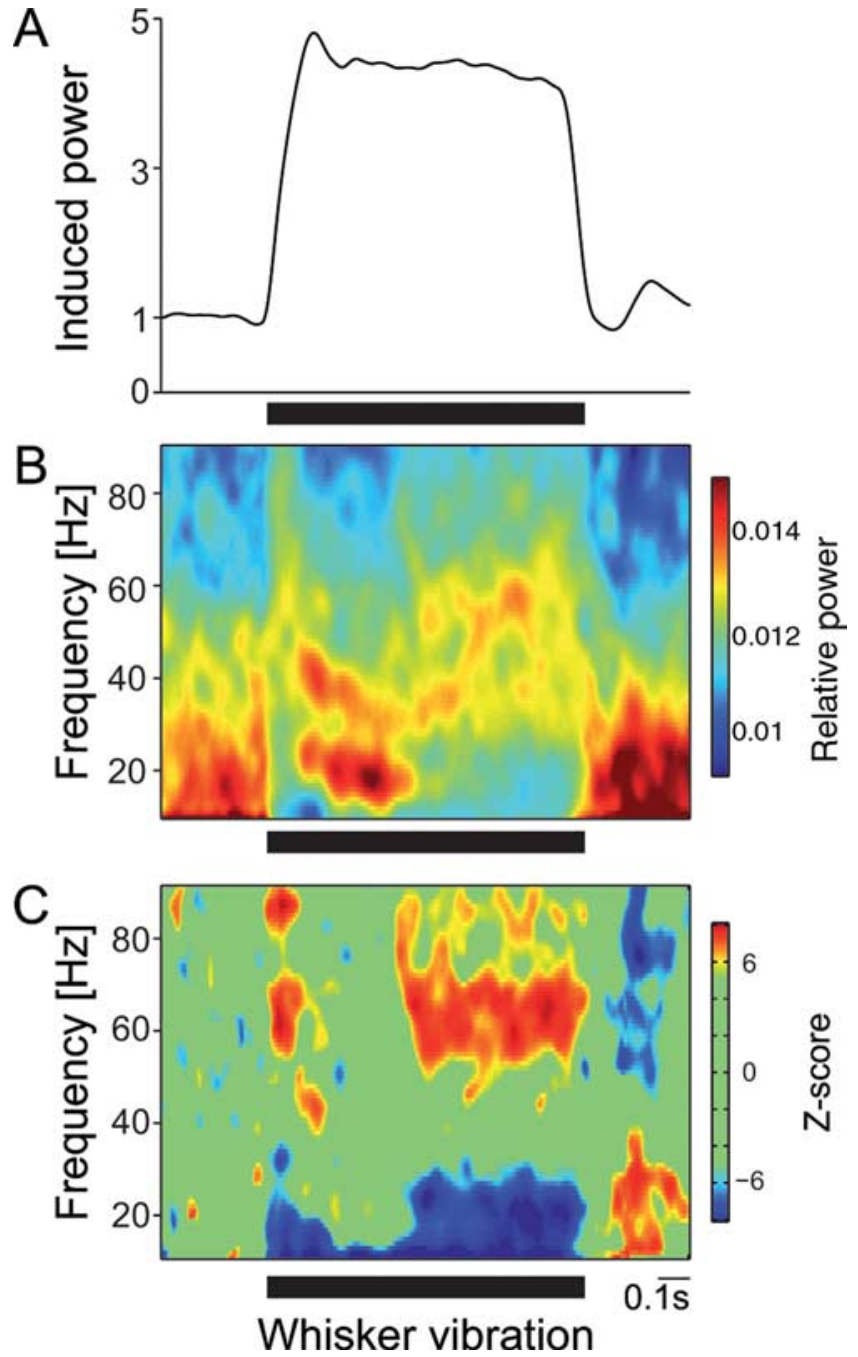

Figure 8. Gamma-band $(30-70 \mathrm{~Hz})$ oscillations induced by high-frequency whisker vibration. Averaged data are shown for a set of 19 PLRs. $\boldsymbol{A}$, The average induced power shows an increase of over fourfold during stimulation. $\boldsymbol{B}$, To compare the oscillation frequencies occurring during stimulation with those during ongoing activity, the relative mean induced power was computed in a time-shifted manner. Although a $1 / f$ frequency distribution is present during ongoing activity, a shift to gamma-band frequencies occurs especially during the second half of the response. $C$, Plot of $z$-scores for the induced response components. Only regions significant at a level of $p \leq 0.01$ (Bonferroni's correction, $z$-score significant at 5.2) are plotted, and all $z$-score values below significance level are colored in green. Black bars mark the stimulation period.

ity has also been obtained in monkey and human somatosensory cortex. In an early study, Ahissar and Vaadia (1990) reported oscillatory single-cell activity over multiple frequency ranges, including the gamma band that was most prominent in ongoing activity but became less stable in frequency for most neurons during tactile stimulation. Lebedev and Nelson (1995) observed gamma oscillations in single units in S1 of monkeys trained to execution of vibratory-cued movements, both in ongoing activity preceding stimulation onset as well as phase locked to the vibratory cue stimulus. Additional studies in the somatosensory system of rats (Nicolelis et al., 1995; Miyashita and Hamada, 1996), cats (Johnson and Alloway, 1994, 1996), and monkeys (Steinmetz et al., 2000) have provided clear evidence for correlated firing of neural populations, suggesting the possibility of assembly formation by neural coherence (Engel et al., 1992, 2001; Singer and Gray, 1995; Herrmann et al., 2004). In humans, several EEG and 
magnetoencephalographic studies have reported task-related augmentation of gamma-band activity in paradigms in which subjects had to direct spatial attention to tactile or painful stimuli applied to a target finger (Desmedt and Tomberg, 1994; Bauer et al., 2007; Hauck et al., 2007).

A key question concerns the possible functional relevance of evoked and induced oscillatory activity in the somatosensory system. Based on the presumed absence of high-frequency phase locking in barrel cortex, models of tactile vibrissal processing have suggested a dual coding strategy (Kleinfeld et al., 2006). Although phase locking was assumed to be relevant for encoding of low-frequency transients occurring during exploratory whisking or of low-frequency vibrations underlying flutter sensation (Salinas et al., 2000), processing of high-frequency input signals during texture discrimination was thought to rely on rate coding rather than on temporal patterning (Kleinfeld et al., 2006). Our data suggest the possibility that evoked response components may also serve for processing of high-frequency temporal stimulation patterns during texture discrimination, as proposed for example by models of closed-loop neural processing (Ahissar and Kleinfeld, 2003).

Currently, the possible functional relevance of induced activity in the somatosensory system is unresolved. In contrast to phase-locked activity, which may be relevant for the encoding of temporal stimulus features, intrinsically generated oscillations might have a function for linking activity distributed across different barrels. This possibility is suggested by studies on the visual system that have demonstrated a role of synchronized induced oscillations for the integration of signals into coherent ensemble activity and for modulating the functional impact of such signals on other neural populations (for review, see Engel et al., 1992, 2001; Singer and Gray, 1995; Herrmann et al., 2004). Testing this possibility for the somatosensory system will require correlation studies that investigate the coherence of induced oscillations as a function of the spatiotemporal configuration of stimulus patterns across the whisker array.

\section{References}

Ahissar E, Kleinfeld D (2003) Closed-loop neuronal computations: focus on vibrissa somatosensation in rat. Cereb Cortex 13:53-62.

Ahissar E, Vaadia E (1990) Oscillatory activity of single units in a somatosensory cortex of an awake monkey and their possible role in texture analysis. Proc Natl Acad Sci USA 87:8935-8939.

Andermann ML, Ritt J, Neimark MA, Moore CI (2004) Neural correlates of vibrissa resonance: band-pass and somatotopic representation of highfrequency stimuli. Neuron 42:451-463.

Andres KH (1966) Über die Feinstruktur der Rezeptoren in Sinushaaren. Z Zellforsch Mikrosk Anat 75:339-365.

Arabzadeh E, Petersen RS, Diamond ME (2003) Encoding of whisker vibration by rat barrel cortex neurons: implications for texture discrimination. J Neurosci 23:9146-9154.

Arabzadeh E, Panzeri S, Diamond ME (2004) Whisker vibration information carried by rat barrel cortex neurons. J Neurosci 24:6011-6020.

Barth DS (2003) Submillisecond synchronization of fast electrical oscillations in neocortex. J Neurosci 23:2502-2510.

Bauer M, Oostenveld R, Peeters M, Fries P (2007) Tactile spatial attention enhances gamma-band activity in somatosensory cortex and reduces lowfrequency activity in parieto-occipital areas. J Neurosci 26:490-501.

Brecht M, Preilowski B, Merzenich MM (1997) Functional architecture of the mystacial vibrissae. Behav Brain Res 84:81-97.

Buhl EH, Tamas G, Fisahn A (1998) Cholinergic activation and tonic excitation induce persistent gamma oscillations in mouse somatosensory cortex in vitro. J Physiol (Lond) 513 1:117-126.

Carvell GE, Simons DJ (1990) Biometric analyses of vibrissal tactile discrimination in the rat. J Neurosci 10:2638-2648.

Carvell GE, Simons DJ (1995) Task- and subject-related differences in sensorimotor behavior during active touch. Somatosens Mot Res 12:1-9.
Chagnac-Amitai Y, Connors BW (1989) Synchronized excitation and inhibition driven by intrinsically bursting neurons in neocortex. J Neurophysiol 62:1149-1162.

Deschênes M, Timofeeva E, Lavallee P (2003) The relay of high-frequency sensory signals in the whisker-to-barreloid pathway. J Neurosci 23:6778-6787.

Desmedt JE, Tomberg C (1994) Transient phase-locking of $40 \mathrm{~Hz}$ electrical oscillations in prefrontal and parietal human cortex reflects the process of conscious somatic perception. Neurosci Lett 168:126-129.

Detsch O, Vahle-Hinz C, Kochs E, Siemers M, Bromm B (1999) Isoflurane induces dose-dependent changes of thalamic somatosensory information transfer. Brain Res 829:77-89.

Detsch O, Kochs E, Siemers M, Bromm B, Vahle-Hinz C (2002) Increased responsiveness of cortical neurons in contrast to thalamic neurons during isoflurane-induced EEG bursts in rats. Neurosci Lett 317:9-12.

Durand D, Greenwood JA (1958) Modifications of the rayleigh test for uniformity in analysis of two-dimensional orientation data. J Geol $66: 229-238$.

Ebara S, Kumamoto K, Matsuura T, Mazurkiewicz JE, Rice FL (2002) Similarities and differences in the innervation of mystacial vibrissal folliclesinus complexes in the rat and cat: a confocal microscopic study. J Comp Neurol 449:103-119.

Engel AK, König P, Kreiter AK, Schillen TB, Singer W (1992) Temporal coding in the visual cortex: new vistas on integration in the nervous system. Trends Neurosci 15:218-226.

Engel AK, Fries P, Singer W (2001) Dynamic predictions: oscillations and synchrony in top-down processing. Nat Rev Neurosci 2:704-716.

Erchova IA, Lebedev MA, Diamond ME (2002) Somatosensory cortical neuronal population activity across states of anaesthesia. Eur J Neurosci 15:744-752.

Friedberg MH, Lee SM, Ebner FF (1999) Modulation of receptive field properties of thalamic somatosensory neurons by the depth of anesthesia. J Neurophysiol 81:2243-2252.

Garabedian CE, Jones SR, Merzenich MM, Dale A, Moore CI (2003) Bandpass response properties of rat SI neurons. J Neurophysiol 90:1379-1391.

Gibson JM, Welker WI (1983) Quantitative studies of stimulus coding in first-order vibrissa afferents of rats. 2. Adaptation and coding of stimulus parameters. Somatosens Res 1:95-117.

Goldberg JM, Brown PB (1969) Response of binaural neurons of dog superior olivary complex to dichotic tonal stimuli: some physiological mechanisms of sound localization. J Neurophysiol 32:613-636.

Gottschaldt KM, Vahle-Hinz C (1981) Merkel cell receptors: structure and transducer function. Science 214:183-186.

Guic-Robles E, Valdivieso C, Guajardo G (1989) Rats can learn a roughness discrimination using only their vibrissal system. Behav Brain Res 31:285-289.

Guic-Robles E, Jenkins WM, Bravo H (1992) Vibrissal roughness discrimination is barrel cortex-dependent. Behav Brain Res 48:145-152.

Hamada Y, Miyashita E, Tanaka H (1999) Gamma-band oscillations in the "barrel cortex" precede rat's exploratory whisking. Neuroscience 88:667-671.

Hara K, Harris RA (2002) The anesthetic mechanism of urethane: the effects on neurotransmitter-gated ion channels. Anesth Analg 94:313-318.

Hartmann MJ, Johnson NJ, Towal RB, Assad C (2003) Mechanical characteristics of rat vibrissae: resonant frequencies and damping in isolated whiskers and in the awake behaving animal. J Neurosci 23:6510-6519.

Harvey MA, Bermejo R, Zeigler HP (2001) Discriminative whisking in the head-fixed rat: optoelectronic monitoring during tactile detection and discrimination tasks. Somatosens Mot Res 18:211-222.

Hauck M, Lorenz J, Engel AK (2007) Attention to painful stimulation enhances $\gamma$-band activity and synchronization in human sensorimotor cortex. J Neurosci 27:9270-9277.

Herrmann CS, Munk MHJ, Engel AK (2004) Cognitive functions of $\gamma$-band activity: memory match and utilization. Trends Cogn Sci 8:347-355.

Johnson MJ, Alloway KD (1994) Sensory modulation of synchronous thalamocortical interactions in the somatosensory system of the rat. Exp Brain Res 102:181-197.

Johnson MJ, Alloway KD (1996) Cross-correlation analysis reveals laminar differences in thalamocortical interactions in the somatosensory system. J Neurophysiol 75:1444-1457.

Jones LM, Depireux DA, Simons DJ, Keller A (2004a) Robust temporal coding in the trigeminal system. Science 304:1986-1989. 
Jones LM, Lee S, Trageser JC, Simons DJ, Keller A (2004b) Precise temporal responses in whisker trigeminal neurons. J Neurophysiol 92:665-668.

Jones MS, Barth DS (1997) Sensory-evoked high-frequency ( $\gamma$-band) oscillating potentials in somatosensory cortex of the unanesthetized rat. Brain Res 768:167-176.

Jones MS, Barth DS (1999) Spatiotemporal organization of fast $(>200 \mathrm{~Hz})$ electrical oscillations in rat vibrissa/barrel cortex. J Neurophysiol 82:1599-1609.

Jones MS, MacDonald KD, Choi B, Dudeck FE, Barth DS (2000) Intracellular correlates of fast $(>200 \mathrm{~Hz})$ electrical oscillations in rat somatosensory cortex. J Neurophysiol 84:1505-1518.

Khatri V, Hartings JA, Simons DJ (2004) Adaptation in thalamic barreloid and cortical barrel neurons to periodic whisker deflections varying in frequency and velocity. J Neurophysiol 92:3244-3254.

Kleinfeld D, Ahissar E, Diamond ME (2006) Active sensation: insights from the rodent vibrissa sensorimotor system. Curr Opin Neurobiol 16:435-444.

Krupa DJ, Matell MS, Brisben AJ, Oliveira LM, Nicolelis MAL (2001) Behavioral properties of the trigeminal somatosensory system in rats performing whisker-dependent tactile discriminations. J Neurosci 21:5752-5763.

Lebedev MA, Nelson RJ (1995) Rhythmically firing $(20-50 \mathrm{~Hz})$ neurons in monkey primary somatosensory cortex: activity patterns during initiation of vibratory-cued hand movements. J Comput Neurosci 2:313-334.

Mehta SB, Kleinfeld D (2004) Frisking the whiskers: patterned sensory input in the rat vibrissa system. Neuron 41:181-184.

Melzer P, Champney GC, Maguire MJ, Ebner FF (2006a) Rate code and temporal code for frequency of whisker stimulation in rat primary and secondary somatic sensory cortex. Exp Brain Res 172:370-386.

Melzer P, Sachdev RNS, Jenkinson N, Ebner FF (2006b) Stimulus frequency processing in awake rat barrel cortex. J Neurosci 26:12198-12205.

Miyashita E, Hamada Y (1996) The "functional connection" of neurones in relation to behavioural states in rats. NeuroReport 7:2407-2411.

Moore CI (2004) Frequency-dependent processing in the vibrissa sensory system. J Neurophysiol 91:2390-2399.

Neimark MA, Andermann ML, Hopfield JJ, Moore CI (2003) Vibrissa resonance as a transduction mechanism for tactile encoding. J Neurosci 23:6499-6509.

Nicolelis MAL, Baccala LA, Lin RCS, Chapin JK (1995) Sensorimotor en- coding by synchronous neural ensemble activity at multiple levels of the somatosensory system. Science 268:1353-1358.

Rice FL, Fundin BT, Arvidsson J, Aldskogius H, Johansson O (1997) Comprehensive immunofluorescence and lectin binding analysis of vibrissal follicle sinus complex innervation in the mystacial pad of the rat. J Comp Neurol 385:149-184.

Ritt JT, Andermann ML, Moore CI (2008) Embodied information processing: vibrissa mechanics and texture features shape micromations in actively sensing rats. Neuron 57:599-613.

Salinas E, Hernandez A, Zainos A, Romo R (2000) Periodicity and firing rate as candidate neural codes for the frequency of vibrotactile stimuli. J Neurosci 20:5503-5515.

Sceniak MP, MacIver MB (2006) Cellular actions of urethane on rat visual cortical neurons in vitro. J Neurophysiol 95:3865-3874.

Shoykhet M, Doherty D, Simons DJ (2000) Coding of deflection velocity and amplitude by whisker primary afferent neurons: implications for higher level processing. Somatosens Mot Res 17:171-180.

Singer W, Gray CM (1995) Visual feature integration and the temporal correlation hypothesis. Annu Rev Neurosci 18:555-586.

Steinmetz PN, Roy A, Fitzgerald PJ, Hsiao SS, Johnson KO, Niebur E (2000) Attention modulates synchronized neuronal firing in primate somatosensory cortex. Nature 404:187-190.

Stüttgen MC, Rüter J, Schwarz C (2006) Two psychophysical channels of whisker deflection in rats align with two neuronal classes of primary afferents. J Neurosci 26:7933-7941.

Tallon-Baudry C, Bertrand O (1999) Oscillatory gamma activity in humans and its role in object representation. Trends Cogn Sci 3:151-162.

Vahle-Hinz C, Gottschaldt KM (1983) Principal differences in the organization of the thalamic face representation in rodents and felids. In: Somatosensory integration in the thalamus (Macchi G, Rustioni A, Spreafico R, eds), pp 125-145. Amsterdam: Elsevier Science.

Vahle-Hinz C, Detsch O, Siemers M, Kochs E, Bromm B (2001) Local $\mathrm{GABA}(\mathrm{A})$ receptor blockade reverses isoflurane's suppressive effects on thalamic neurons in vivo. Anesth Analg 92:1578-1584.

Vahle-Hinz C, Detsch O, Siemers M, Kochs E (2007) Contributions of GABAergic and glutamatergic mechanisms to isoflurane-induced suppression of thalamic somatosensory information transfer. Exp Brain Res 176:159-172. 